\title{
On Discretizations of the Vector Nonlinear Schrödinger Equation
}

\author{
Mark J. Ablowitz \\ Department of Applied Mathematics \\ University of Colorado-Boulder \\ Boulder, Colorado, 80309, USA \\ Yasuhiro Ohta \\ Department of Applied Mathematics, \\ Faculty of Engineering, Hiroshima University \\ 1-4-1 Kagamiyama, Higashi-Hiroshima 739-8527, Japan \\ A. David Trubatch \\ Department of Applied Mathematics \\ University of Colorado-Boulder \\ Boulder, Colorado, 80309, USA
}

\begin{abstract}
Two discretizations of the vector nonlinear Schrödinger (NLS) equation are studied. One of these discretizations, referred to as the symmetric system, is a natural vector extension of the scalar integrable discrete NLS equation. The other discretization, referred to as the asymmetric system, has an associated linear scattering pair. General formulae for soliton solutions of the asymmetric system are presented. Formulae for a constrained class of solutions of the symmetric system may be obtained. Numerical studies support the hypothesis that the symmetric system has general soliton solutions.
\end{abstract}

PACS Numbers: 03.40.Kf, 46.10+z, 42.81.Dp, 42.81.Gs

\section{Introduction}

In recent years there has been wide interest in the study of solitons and integrable systems. Researchers have found that not only are continuous systems (i.e. PDE's) integrable via the inverse scattering transform (IST) but also that interesting classes of discrete systems (semi-discrete as well as partial difference equation) are integrable - cf. Ablowitz and Segur 11 for an early review). In this letter, we discuss soliton solutions and the integrable nature of certain discrete systems associated with the vector extensions of the nonlinear Schrödinger (NLS) equation:

$$
i q_{t}=q_{x x}+2 q|q|^{2} .
$$

NLS is a centrally important and physically significant nonlinear equation which possesses solitons and is integrable via IST [2]. Furthermore, NLS arises in many areas of physics, such as the evolution of small amplitude slowly varying wave packets in: deep water, nonlinear optics and plasma physics (see e.g. [1]).

In 1974, Manakov [3] showed that the vector NLS (VNLS),

$$
i \mathbf{q}_{t}=\mathbf{q}_{x x}+2\|\mathbf{q}\|^{2} \mathbf{q}
$$

where $\mathbf{q}$ is an $N$-component vector and $\|\cdot\|$ denotes the vector norm, also possessed solitons and could be integrated via IST (actually, in [3] only the case $N=2$ was studied in detail; however the extension to the 
$N$-th order vector system is straightforward). The second order $(N=2)$ VNLS equation is relevant in the study of electromagnetic waves in optical media in which the electric field has two nontrivial components. In optical fibers, the components of $\mathbf{q}$ in eq. (2) correspond to components of the electric field transverse to the direction of wave propagation. These components of the transverse field compose a basis of the polarization states. Although, in optical fibers, the equations governing the field are in general a non-integrable variation of vector NLS [4], there are circumstances in which eq. (2) is the appropriate model [5, 6].

In 1976, Ablowitz and Ladik [7] found that the following discrete system is integrable via IST:

$$
i \frac{\mathrm{d}}{\mathrm{d} t} q_{n}=\frac{1}{h^{2}}\left(q_{n-1}-2 q_{n}+q_{n+1}\right)+\left|q_{n}\right|^{2}\left(q_{n+1}+q_{n-1}\right)
$$

The continuum $(h \rightarrow 0)$ limit of this discrete system (3) is NLS (11) hence this system can be referred to as integrable discrete NLS (IDNLS). The discrete system itself is also useful in physical applications (see e.g. [8, 9, 10, 11]).

IDNLS provides an excellent numerical scheme to solve NLS (11) (cf. [12]). Computations demonstrate that, by simply replacing the nonlinear term in (3) with $2\left|q_{n}\right|^{2} q_{n}$, one obtains a poor numerical scheme. In contrast, the preservation of the integrable structure in IDNLS (3) plays a key role in its utility as a numerical scheme. This points out that care must be taken in the choice of discretization of the nonlinear terms for the purpose of numerical simulations.

It is natural to look for useful discretizations of VNLS (2). However, the special character of IDNLS as compared to other discretizations of NLS, suggests that different discretizations of VNLS may have very different dynamics. Therefore, the choice of discretization merits close analysis.

In this letter, we discuss two discretizations of VNLS which we refer to as the symmetric and asymmetric discretizations. These discretizations are as follows:

Symmetric discretization:

$$
i \frac{\mathrm{d}}{\mathrm{d} t} \mathbf{q}_{n}=\frac{1}{h^{2}}\left(\mathbf{q}_{n-1}-2 \mathbf{q}_{n}+\mathbf{q}_{n+1}\right)+\left\|\mathbf{q}_{n}\right\|^{2}\left(\mathbf{q}_{n-1}+\mathbf{q}_{n+1}\right)
$$

where $\mathbf{q}_{n}$ is an $N$-component vector.

Asymmetric discretization:

$$
\begin{aligned}
i \frac{\mathrm{d}}{\mathrm{d} t} \mathbf{q}_{n} & =\frac{1}{h^{2}}\left(\mathbf{q}_{n-1}-2 \mathbf{q}_{n}+\mathbf{q}_{n+1}\right)-\left(\mathbf{r}_{n}^{T} \mathbf{q}_{n-1}\right) \mathbf{q}_{n}-\left(\mathbf{r}_{n}^{T} \mathbf{q}_{n}\right) \mathbf{q}_{n+1} \\
-i \frac{\mathrm{d}}{\mathrm{d} t} \mathbf{r}_{n} & =\frac{1}{h^{2}}\left(\mathbf{r}_{n-1}-2 \mathbf{r}_{n}+\mathbf{r}_{n+1}\right)-\left(\mathbf{r}_{n}^{T} \mathbf{q}_{n}\right) \mathbf{r}_{n-1}-\left(\mathbf{r}_{n+1}^{T} \mathbf{q}_{n}\right) \mathbf{r}_{n}
\end{aligned}
$$

where, as before, $\mathbf{q}_{n}$ is an $N$-component vector as is $\mathbf{r}_{n}$. The superscript $T$ denotes the transpose.

Both systems (4, $5 \mathrm{a} / 5 \mathrm{~b})$ reduce to VNLS (2) in the continuum limit $(h \rightarrow 0)$ where in $(5 \mathrm{a}-5 \mathrm{~b})$ we take $\mathbf{r}=-\mathbf{q}^{*}$, where $*$ denotes the complex conjugate, after taking the continuum limit. The asymmetric system (5a 5b), however, does not admit the symmetry $\mathbf{r}_{n}=-\mathbf{q}_{n}^{*}$ and hence remains "asymmetric" for $h$ finite.

These systems have a number of interesting properties. Both systems (4, 5a $5 \mathrm{~b}$ ) reduce to the integrable discrete scalar case (3), just as VNLS (2) reduces to NLS (11). The asymmetric system (5a $5 \mathrm{~b}$ ) is associated with a linear operator pair (see eq. 11a 11b). Although there is no known linear operator pair for the symmetric system (何), under the reduction $\mathbf{q}_{n}=e^{i \omega t} \mathbf{v}_{n}$, where $\mathbf{v}_{n}$ is independent of $t$, this equation reduces to an $N$-dimensional difference equation which is known to be integrable (cf. [13).

Both the symmetric (4) and asymmetric (5a-5b) systems possess a class of soliton solutions. The asymmetric system has a class of soliton solutions which correspond to all the soliton solutions of the continuum limit NLS (2). However, the exact multi-soliton solutions found so far for the symmetric system (位) only reduce to a subset of those associated with eq. (2). Since the known exact soliton solutions of eq. (4) are only a subset of those known in the continuum limit (2), we numerically examined the interactions of solitary waves associated with the symmetric system which lie outside this class. The numerical evidence indicates that, in the symmetric system, the solitary waves interact elastically and are therefore true solitons. This finding supports the possibility that the symmetric system (14) is indeed integrable. 


\section{NLS, Integrable discrete NLS and Vector NLS}

We briefly review the relationship between NLS, IDNLS and Vector NLS in order to clarify their relation to the symmetric and asymmetric discretizations of vector NLS.

\subsection{NLS and IDNLS}

NLS (II) can be obtained as compatibility condition of a linear operator pair (e.g. a Lax pair). The linear pair can be used to solve (1i) by IST. The existence of an associated linear operator pair and IST are hallmarks of integrability. Therefore, in the effort to construct an integrable discretization of NLS, it is natural to look for a discrete version of the operator pair and the compatibility condition. In fact, the integrable discretization of NLS (3) was obtained in exactly this manner [7].

The extent to which the integrable structure of NLS is preserved under the integrable discretization is significant: The IST for IDNLS is analogous to the theory for NLS. In particular, the one-soliton solution of IDNLS (3) given by

$$
q_{n}(t)=A e^{-i(b h n-\omega t-\phi)} \operatorname{sech}(a h n-v t-\theta)=e^{i \phi} \hat{q}_{n}(t)
$$

with

$$
A=\frac{\sinh (a h)}{h}, \quad \omega=\frac{2(1-\cosh (a h) \cos (b h))}{h^{2}}, \quad v=2 \frac{\sinh (a h) \sin (b h)}{h^{2}}
$$

where $a, b, \theta, \phi$ are arbitrary parameters, converges (as $h \rightarrow 0$ ) to the one-soliton solution of NLS with the same four free parameters. More generally, solutions of IDNLS converge to the solutions of NLS with an error of $O\left(h^{2}\right)$.

For both NLS and IDNLS, generic rapidly-decaying initial data resolves itself into some number solitons plus radiation that vanishes as $t \rightarrow \infty$ (in the sup norm). Even though the solitons interact nonlinearly upon collision, it is well-known that they retain their shape and speed after the collision. This can be determined by comparing the forward $(t \rightarrow+\infty)$ and backward $(t \rightarrow-\infty)$ long-time limits of the solutions. In these long-time limits, solitons traveling at different speeds are well-separated. Although this collision is elastic, the position and overall complex phase of the individual solitons are shifted between the forward and backwards long-time limits. This elastic interaction of solitons up to a shift in phase (position and overall complex phase) is typical of $(1+1)$-dimensional integrable systems.

\section{$2.2 \quad$ VNLS}

VNLS (2) can be obtained by substituting the vector $\mathbf{q}$ for $q$ in NLS (1D) and replacing complex conjugation with the Hermitian conjugate. Similarly, the linear operator pair for VNLS can be obtained by appropriately making the same substitution of scalars by vectors in the linear operator pair for NLS (cf. Manakov [3]).

When considered as a model for the propagation of electromagnetic waves in optical fibers, the components of VNLS play the role of a basis for the polarization vector. In the derivation of VNLS by Manakov [3] the choice of basis is arbitrary. Therefore, the vector system (2) ought to be, and is, invariant under a change of basis for the polarization. Mathematically, a change of basis is obtained by multiplying the independent variable $\mathbf{q}$ by a unitary matrix, $\mathbf{U}$. Therefore, the freedom in the choice of basis is reflected in the fact that the vector system (2) is invariant under the transformation $\mathbf{q} \rightarrow \mathbf{U q}$. This symmetry is an important feature in distinguishing among discrete versions of vector NLS (notably, both the versions of discrete vector NLS discussed in this letter (4.5 5 b retain this symmetry).

Under the reduction

$$
\mathbf{q}=\mathbf{c} \hat{q}
$$

where $\mathbf{c}$ is a constant, $N$-component vector such that $\|\mathbf{c}\|^{2}=1$ and $\hat{q}$ is a scalar function of $x$ and $t$, VNLS (2) reduces to NLS (11). This is a manifestation of the fact that the vector system is a generalization of 
NLS obtained by allowing the polarization to be non-constant. When the solution of VNLS has a constant polarization, NLS is recovered. As a consequence, any solution of scalar NLS has a corresponding family of solutions of VNLS. We call a solution of the form (7) a reduction solution. Because the vector $\mathbf{c}$ is arbitrary (up to the constraint that it is of unit length), a reduction solution has correspondingly more parameters than the solution of scalar NLS to which it reduces. We refer to the vector $\mathbf{c}$ in eq. (7) as the polarization of the reduction solution.

The soliton solutions of vector NLS can be found by IST [3]. The one-soliton solution of vector NLS is the reduction solution (i.e of the form (7) where $q_{n}$ is the one-soliton solution of NLS. As in the scalar case, the IST [3] shows that solitons interact elastically. By elastic, it is meant that the solitons retain their shape and speed after interaction as shown by comparing the forward and backward long-time limits.

For the vector system, care is needed: the nature of the phase shift for vector solitons is somewhat different than the scalar case. In addition to a shift in the center of the peak (as in the scalar case), vector solitons also undergo a change in polarization upon collision. That is, in the forward and backward long-time limit, the solution asymptotically approaches a linear superposition of the individual solitons:

$$
\mathbf{q} \sim \sum_{j} \mathbf{q}_{j}^{ \pm} \quad \text { as } \quad t \rightarrow \pm \infty
$$

where $\mathbf{q}_{j}^{ \pm}=\mathbf{c}_{j}^{ \pm} \hat{q}_{j}^{ \pm}$and, for each $j, \hat{q}_{j}^{+}$and $\hat{q}_{j}^{-}$are one-soliton solutions of NLS with the same amplitude and speed. Comparison of the forward $(+)$ and backward $(-)$ long-time limits shows that

$$
\mathbf{c}_{j}^{-} \neq \mathbf{c}_{j}^{+},
$$

but

$$
\left\|\mathbf{c}_{j}^{+}\right\|^{2}=\left\|\mathbf{c}_{j}^{-}\right\|^{2}=1 .
$$

A closed formula for this shift in the polarization can be calculated by considering the eigenfunction of the associated scattering problem [3].

We refer to the squared modulus of a component of the polarization vector as the intensity of that component of the polarization. That is, if $c_{j}^{(\ell)}$ is the $\ell$-th component of $\mathbf{c}_{j}$, the polarization vector of the soliton $j$, then the intensity of the of the $\ell$-th component of soliton $j$ is $\left|c_{j}^{(\ell)}\right|^{2}$. The relation (8) implies that the total intensity of each soliton is preserved, specifically the sum of the intensities is equal to one. However, the distribution of intensity between the components of the polarization of an individual soliton will not, in general, be equal in the forward and backwards long-time limits due to interaction with other solitons: subject to the constraint of eq. (8), in general,

$$
\left|c_{j}^{(\ell)-}\right|^{2} \neq\left|c_{j}^{(\ell)+}\right|^{2} .
$$

This change in the distribution of intensity is a distinctive feature of the vector system. There is no corresponding phenomenon in the soliton interactions of the scalar equation (in both the scalar and vector equations, the location of the peak of an individual soliton is shifted by the soliton interaction).

Manakov [3] also observed the following special case for the interaction of the solitons of VNLS: If, for every pair of solitons $j$ and $k$, either

$$
\left|\mathbf{c}_{j}^{-} \cdot \mathbf{c}_{k}^{-}\right|=0
$$

or

$$
\left|\mathbf{c}_{j}^{-} \cdot \mathbf{c}_{k}^{-}\right|=1
$$


where $\cdot$ denotes the dot product, then

$$
\left|\mathbf{c}_{j}^{-} \cdot \mathbf{c}_{j}^{+}\right|=1
$$

for all $j$. As a consequence of (10), $\left|c_{j}^{(\ell)-}\right|^{2}=\left|c_{j}^{(\ell)+}\right|^{2}$ for all $j, \ell$. Physically, this means that, in any polarization basis, the distribution of intensity among the components of polarization for an individual soliton is not changed by the soliton interaction.

\section{Asymmetric Discrete Vector NLS}

The preceding derivations of IDNLS and VNLS from NLS suggest two methods to obtain an operator pair which has a discrete form of VNLS as its compatibility condition: (i) discretize the operator pair for vector NLS in a manner analogous to that used to obtain the operator pair of IDNLS or (ii) appropriately substitute vectors in to the operator pair for IDNLS. These approaches yield the linear operator pair

$$
\begin{aligned}
\mathbf{S}_{n} & =\left(\begin{array}{cc}
z \mathbf{I}_{N} & h \mathbf{q}_{n} \\
h \mathbf{r}_{n}^{T} & \frac{1}{z}
\end{array}\right) \\
\mathbf{T}_{n} & =\left(\begin{array}{cc}
i \mathbf{q}_{n} \mathbf{r}_{n-1}^{T}-i \frac{1}{2 h^{2}}\left(z-\frac{1}{z}\right)^{2} \mathbf{I}_{N} & -i \frac{1}{h}\left(\frac{1}{z} \mathbf{q}_{n}-z \mathbf{q}_{n-1}\right) \\
i \frac{1}{h}\left(\frac{1}{z} \mathbf{r}_{n}^{T}-z \mathbf{r}_{n-1}^{T}\right) & -i \mathbf{r}_{n}^{T} \mathbf{q}_{n-1}+i \frac{1}{2 h^{2}}\left(z-\frac{1}{z}\right)^{2}
\end{array}\right)
\end{aligned}
$$

where $\mathbf{I}_{N}$ is the $N \times N$ identity matrix. The asymmetric system (5a $5 \mathrm{~b}$ ) is the compatibility condition of this pair.

Under the reduction

$$
\mathbf{q}_{n}=\mathbf{c} \hat{q}_{n}, \quad \mathbf{r}_{n}=\mathbf{d} \hat{q}_{n}^{*}
$$

where $\mathbf{c}$ and $\mathbf{d}$ are constant vectors such that $\mathbf{c} \cdot \mathbf{d}=1$ and $\hat{q}_{n}$ is a scalar function of $n$ and $t$, the asymmetric system (5a 5b) reduces to IDNLS (3). Solutions of this form (12) are referred to as reduction solutions. Hence, every solution of IDNLS generates a family of reduction solutions of the asymmetric system. We are particularly interested in reduction solutions where $\mathbf{d}=-\mathbf{c}^{*}$ because, in this case, $\mathbf{r}_{n}=-\mathbf{q}_{n}^{*}$ and (5a-5b) reduces to a single equation.

\subsection{General Formula for Multi-soliton Solutions}

Multi-soliton solutions of the asymmetric system can be derived, without the use of the IST machinery, by Hirota's method. First, make the independent-variable transformations

$$
\mathbf{q}_{n}=\frac{\mathbf{g}_{n}}{f_{n}}, \quad \mathbf{r}_{n}=\frac{\overline{\mathbf{g}}_{n}}{f_{n}}
$$

where $\mathbf{g}_{n}$ and $\overline{\mathbf{g}}_{n}$ are vectors with $N$ components and $f_{n}$ is a scalar. Then, solutions of the system of bilinear equations

$$
\begin{gathered}
f_{n+1} f_{n-1}-f_{n}^{2}=\overline{\mathbf{g}}_{n}^{T} \mathbf{g}_{n} \\
i h^{2} D_{t} f_{n} \cdot \mathbf{g}_{n}=f_{n-1} \mathbf{g}_{n+1}-2 f_{n} \mathbf{g}_{n}+f_{n+1} \mathbf{g}_{n-1}-\mathbf{H}_{n} \overline{\mathbf{g}}_{n} \\
-i h^{2} D_{t} f_{n} \cdot \overline{\mathbf{g}}_{n}=f_{n-1} \overline{\mathbf{g}}_{n+1}-2 f_{n} \overline{\mathbf{g}}_{n}+f_{n-1} \overline{\mathbf{g}}_{n+1}-\overline{\mathbf{H}}_{n} \mathbf{g}_{n} \\
f_{n} \mathbf{H}_{n+1}=h^{2}\left(\mathbf{g}_{n} \mathbf{g}_{n+1}^{T}-\mathbf{g}_{n+1} \mathbf{g}_{n}^{T}\right) \\
f_{n} \overline{\mathbf{H}}_{n-1}=h^{2}\left(\overline{\mathbf{g}}_{n-1} \overline{\mathbf{g}}_{n}^{T}-\overline{\mathbf{g}}_{n} \overline{\mathbf{g}}_{n-1}^{T}\right)
\end{gathered}
$$

where the $N \times N$ matrices $\mathbf{H}_{n}$ and $\overline{\mathbf{H}}_{n}$ are auxiliary variables, are solutions of the asymmetric system (5a) 5b). 
The $M$-soliton solutions for these bilinear equations (13a 130 are given by the following determinants:

$$
\begin{aligned}
& f_{n}=\left|\begin{array}{cc}
\mathbf{A}_{n} & \mathbf{I} \\
-\mathbf{I} & \mathbf{B}
\end{array}\right|, \quad g_{n}^{(k)}=\left|\begin{array}{ccc}
\mathbf{A}_{n} & \mathbf{I} & \Phi_{n} \\
-\mathbf{I} & \mathbf{B} & 0 \\
0 & \mathbf{\Psi}_{k} & 0
\end{array}\right|, \quad \bar{g}_{n}^{(k)}=\left|\begin{array}{ccc}
\mathbf{A}_{n} & \mathbf{I} & 0 \\
-\mathbf{I} & \mathbf{B} & \overline{\mathbf{\Psi}}_{k} \\
\overline{\mathbf{\Phi}}_{n} & 0 & 0
\end{array}\right| \\
& H_{n}^{(\ell, j)}=\left|\begin{array}{cccc}
\mathbf{A}_{n} & \mathbf{I} & \mathbf{\Phi}_{n-1} & \mathbf{\Phi}_{n} \\
-\mathbf{I} & \mathbf{B} & 0 & 0 \\
0 & \mathbf{\Psi}_{\ell} & 0 & 0 \\
0 & \boldsymbol{\Psi}_{j} & 0 & 0
\end{array}\right|, \quad \bar{H}_{n}^{(\ell, j)}=\left|\begin{array}{cccc}
\mathbf{A}_{n} & \mathbf{I} & 0 & 0 \\
-\mathbf{I} & \mathbf{B} & \overline{\mathbf{\Psi}}_{\ell} & \overline{\mathbf{\Psi}}_{j} \\
\overline{\mathbf{\Phi}}_{n} & 0 & 0 & 0 \\
\overline{\mathbf{\Phi}}_{n+1} & 0 & 0 & 0
\end{array}\right|
\end{aligned}
$$

where: $\mathbf{I}$ is the $M \times M$ identity matrix;

(i) $\mathbf{A}_{n}$ and $\mathbf{B}$ are $M \times M$ matrices defined by

$$
A_{n}^{(\ell, j)}=\frac{h}{e^{h\left(p_{\ell}+p_{j}^{*}\right)}-1} e^{\eta_{\ell, n}+\eta_{j, n}^{*}}, \quad B^{(\ell, j)}=\frac{h}{e^{h\left(p_{\ell}^{*}+p_{j}\right)}-1} \gamma_{\ell}^{H} \gamma_{j}
$$

with

$$
\eta_{j, n}=p_{j} n h+\frac{i}{h^{2}}\left(2-e^{h p_{j}}-e^{-h p_{j}}\right) t
$$

the complex numbers $p_{j}=a_{j}-i b_{j}, a_{j}>0$, determine the amplitude and speed of the $j$-th soliton; the $N$-component complex vectors $\gamma_{j}$ and $\bar{\gamma}_{j}(j=1, \ldots, M)$ determine the polarizations and envelope phases of the solitons;

(ii) $\boldsymbol{\Phi}_{n}, \overline{\mathbf{\Phi}}_{n}$ are, respectively, the $M$-component column and row vectors

$$
\Phi_{n}^{(\ell)}=e^{-h p_{\ell}} e^{\eta_{\ell, n}}, \quad \bar{\Phi}_{n}^{(j)}=e^{\eta_{j, n}^{*}}
$$

(iii) $\boldsymbol{\Psi}_{k}, \overline{\mathbf{\Psi}}_{k}$ are, respectively, the $M$-component row and column vectors,

$$
\Psi_{k}^{(j)}=-\gamma_{j}^{(k)}, \quad \bar{\Psi}_{k}^{(\ell)}=e^{-h p_{\ell}^{*}} \bar{\gamma}_{\ell}^{(k)}
$$

\subsection{The One-Soliton Solution}

From the above formulae $14 \mathrm{a}-14 \mathrm{~b})$, the one-soliton $(M=1)$ solution is

$$
\begin{aligned}
& \mathbf{q}_{n}=\frac{\gamma_{1}}{\left(\bar{\gamma}_{1}^{T} \gamma_{1}\right)^{\frac{1}{2}}} e^{-i h b_{1}} \frac{\sinh \left(a_{1} h\right)}{h} e^{i \beta_{1, n}} \operatorname{sech}\left(\alpha_{1, n}+\delta_{1}\right) \\
& \mathbf{r}_{n}=-\frac{\gamma_{1}}{\left(\bar{\gamma}_{1}^{T} \gamma_{1}\right)^{\frac{1}{2}}} e^{-i h b_{1}} \frac{\sinh \left(a_{1} h\right)}{h} e^{-i \beta_{1, n}} \operatorname{sech}\left(\alpha_{1, n}+\delta_{1}\right)
\end{aligned}
$$

where

$$
\begin{aligned}
& \alpha_{1, n}=\operatorname{Re} \eta_{1, n}=a_{1} h n-2 \frac{\sinh \left(a_{1} h\right) \sin \left(b_{1} h\right)}{h^{2}} t \\
& \beta_{1, n}=\operatorname{Im} \eta_{1, n}=-b_{1} h n+\frac{2\left(1-\cosh \left(a_{1} h\right) \cos \left(b_{1} h\right)\right)}{h^{2}}
\end{aligned}
$$


and

$$
\delta_{1}=\log \frac{1}{2}\left\{\bar{\gamma}_{1}^{T} \gamma_{1}\right\}-\log \left\{\frac{e^{2 a_{1} h}-1}{h}\right\} .
$$

Note that to get a solution $(16 \mathrm{a}-16 \mathrm{~b})$ with $\delta_{1}$ real we restrict our attention to the case where $\bar{\gamma}_{1}^{T} \gamma_{1}$ is real and positive. The one-soliton solution (16a $16 \mathrm{~b}$ ) is a reduction solution-i. e. of the form (12) -where

$$
\mathbf{c}=\frac{\gamma_{1}}{\left(\overline{\boldsymbol{\gamma}}_{1}^{T} \boldsymbol{\gamma}_{1}\right)^{\frac{1}{2}}} e^{-i h b_{1}}, \quad \mathbf{d}=-\frac{\bar{\gamma}_{1}}{\left(\overline{\boldsymbol{\gamma}}_{1}^{T} \boldsymbol{\gamma}_{1}\right)^{\frac{1}{2}}} e^{i h b_{1}}
$$

and the scalar function $\hat{q}_{n}$ is given by eq. (6) - the one-soliton solution of IDNLS -where $a=a_{1}, b=b_{1}$ and $\theta=-\delta_{1}$. In this solution, $\mathbf{d}=-\mathbf{c}^{*}$ if, and only if, $\bar{\gamma}_{1}=\gamma_{1}^{*}$.

\subsection{Two soliton Interaction}

To show that, for $M>1$, the determinants (14a-14b) indeed give a multi-soliton solution, we consider the long-time limits. In these limits, solitons moving at different speeds are separated. For concreteness, let $M=2$ and

$$
\sinh \left(a_{1} h\right) \sin \left(b_{1} h\right)>\sinh \left(a_{2} h\right) \sin \left(b_{2} h\right)
$$

for the given $a_{1}, b_{1}, a_{2}, b_{2}$. The condition (18) ensures that each soliton travels with a different speed (the analysis below holds, in general for $M$ solitons as long as each has a different speed). When the solitons travel at different speeds, we determine the asymptotic form of an individual soliton by taking long-time limits in a coordinate frame moving with that soliton.

The limits

$$
n \sim 2 \frac{\sinh \left(a_{j} h\right) \sin \left(b_{j} h\right)}{a_{j} h^{3}} t, \quad t \rightarrow \pm \infty
$$

are long-time limits in a coordinate frame moving with soliton $j$. In the limit (19) with $j=1$

$$
\operatorname{Re} \eta_{1, n}=\text { const. } \quad \text { and } \quad \operatorname{Re} \eta_{2, n} \rightarrow \pm \infty \quad \text { as } \quad t \rightarrow \pm \infty
$$

The substitution of (20) into eq. (14a $14 \mathrm{~b})$, where $M=2$, yields

$$
\mathbf{q}_{n} \rightarrow \mathbf{q}_{1, n}^{ \pm} \quad \text { and } \quad \mathbf{r}_{n} \rightarrow \mathbf{r}_{1, n}^{ \pm} \quad \text { as } \quad t \rightarrow \pm \infty,
$$

which is the asymptotic form of soliton 1 in the forward $(+)$ and backward $(-)$ long-time limits. The longtime limits for soliton 2 are similar. The coordinate frame of soliton 2 is obtained by the the limit (19) with $j=2$ which yields

$$
\text { Re } \eta_{1, n} \rightarrow \mp \infty \quad \text { and } \quad \operatorname{Re} \eta_{2, n}=\text { const } \quad \text { as } \quad t \rightarrow \pm \infty
$$

Note the change in relative sign between $\eta_{1, n}$ and $t$ in the coordinate frame of soliton 2 - the slower soliton -in the long-time limit. To obtain

$$
\mathbf{q}_{n} \rightarrow \mathbf{q}_{2, n}^{ \pm} \quad \text { and } \quad \mathbf{r}_{n} \rightarrow \mathbf{r}_{2, n}^{ \pm} \quad \text { as } \quad t \rightarrow \pm \infty
$$


which is the asymptotic form of soliton 2 in the forward $(+)$ and backward $(-)$ long-time limits, substitute (22) into (14a-14b), where $M=2$. Combining (21) and (23) gives

$$
\mathbf{q}_{n} \sim \mathbf{q}_{1, n}^{ \pm}+\mathbf{q}_{2, n}^{ \pm} \quad \text { and } \quad \mathbf{r}_{n} \sim \mathbf{r}_{1, n}^{ \pm}+\mathbf{r}_{2, n}^{ \pm} \quad \text { as } \quad t \rightarrow \pm \infty .
$$

The effect of interaction on the solitons is determined by comparing $\mathbf{q}_{j, n}^{-}$and $\mathbf{r}_{j, n}^{-}$with $\mathbf{q}_{j, n}^{+}$and $\mathbf{r}_{j, n}^{+}$for $j=1,2$. We give the formulae for the asymptotic forms of the solitons below.

First, we consider soliton 1. In the backward long-time limit,

$$
\mathbf{q}_{1, n}^{-}=\mathbf{c}_{1}^{-} \hat{q}_{1, n}^{-}, \quad \mathbf{r}_{1, n}^{-}=\mathbf{d}_{1}^{-} \hat{q}_{1, n}^{-*}
$$

where

$$
\begin{aligned}
\mathbf{c}_{1}^{-} & =\frac{\gamma_{1}}{\left(\bar{\gamma}_{1}^{T} \gamma_{1}\right)^{\frac{1}{2}}} e^{-i h b_{1}} \\
\mathbf{d}_{1}^{-} & =-\frac{\bar{\gamma}_{1}}{\left(\bar{\gamma}_{1}^{T} \gamma_{1}\right)^{\frac{1}{2}}} e^{i h b_{1}}
\end{aligned}
$$

and $\hat{q}_{1, n}^{-}$is a one-soliton solution of IDNLS (the $\hat{q}$ in eq. (6)) with $a=a_{1}, b=b_{1}$ and

$$
\theta=-\delta_{1}^{-}=-\log \frac{1}{2}\left\{\overline{\boldsymbol{\gamma}}_{1}^{T} \boldsymbol{\gamma}_{1}\right\}+\log \left\{\frac{e^{2 a_{1} h}-1}{h}\right\} .
$$

As in the one-soliton case, we require that $\bar{\gamma}_{1}^{T} \gamma_{1}$ is real and positive. The forward long-time for soliton 1 is

$$
\mathbf{q}_{1, n}^{+}=\mathbf{c}_{1}^{+} \hat{q}_{1, n}^{+} \quad \mathbf{r}_{1, n}^{+}=\mathbf{d}^{+} \hat{q}_{1, n}^{+*}
$$

where

$$
\begin{aligned}
& \mathbf{c}_{1}^{+}=\frac{1}{\chi}\left(e^{-h p_{1}} \sigma_{2}-\rho^{*}\right)\left(\sigma_{2}\left(\overline{\boldsymbol{\gamma}}_{2}^{T} \boldsymbol{\gamma}_{2}\right) \boldsymbol{\gamma}_{1}-\rho^{*}\left(\overline{\boldsymbol{\gamma}}_{2}^{T} \boldsymbol{\gamma}_{1}\right) \boldsymbol{\gamma}_{2}\right) \\
& \mathbf{d}_{1}^{+}=-\frac{1}{\chi} e^{-h p_{1}^{*}}\left(\sigma_{2}-\rho\right)\left(\sigma_{2}\left(\overline{\boldsymbol{\gamma}}_{2}^{T} \boldsymbol{\gamma}_{2}\right) \overline{\boldsymbol{\gamma}}_{1}-\rho\left(\boldsymbol{\gamma}_{2}^{T} \overline{\boldsymbol{\gamma}}_{1}\right) \overline{\boldsymbol{\gamma}}_{2}\right)
\end{aligned}
$$

and $\hat{q}_{1, n}^{+}$is a one soliton solution of IDNLS with $a=a_{1}, b=b_{1}$ and

$$
\theta=-\delta_{1}^{+}=-\log \chi+\log \sigma_{2}+\log \left\{\overline{\boldsymbol{\gamma}}_{2}^{T} \boldsymbol{\gamma}_{2}\right\}
$$

also

$$
\chi=\left\{\left(\sigma_{1} \sigma_{2}-|\rho|^{2}\right)\left(\sigma_{1} \sigma_{2}\left(\bar{\gamma}_{1}^{T} \gamma_{1}\right)\left(\bar{\gamma}_{2}^{T} \gamma_{2}\right)-|\rho|^{2}\left(\bar{\gamma}_{1}^{T} \boldsymbol{\gamma}_{2}\right)\left(\overline{\boldsymbol{\gamma}}_{2}^{T} \boldsymbol{\gamma}_{1}\right)\right)\right\}^{\frac{1}{2}}
$$

and we require that $\gamma_{j}, \bar{\gamma}_{j}$ for $j=1,2$ are such that $\chi$ and $\bar{\gamma}_{2}^{T} \gamma_{2}$ are real and positive. The constants $\sigma_{1}$, $\sigma_{2}, \rho$ are given by

$$
\sigma_{1}=\frac{h}{e^{h\left(p_{1}+p_{1}^{*}\right)}-1}, \quad \sigma_{2}=\frac{h}{e^{h\left(p_{2}+p_{2}^{*}\right)}-1}, \quad \rho=\frac{h}{e^{h\left(p_{1}+p_{2}^{*}\right)}-1} .
$$


Hence, the expression $\left(\sigma_{1} \sigma_{2}-|\rho|^{2}\right)$ is real and positive. Note that, although $\mathbf{c}_{1}^{-} \neq \mathbf{c}_{1}^{+}, \mathbf{d}_{1}^{-} \neq \mathbf{d}_{1}^{+}$, and $\delta_{1}^{-} \neq \delta_{1}^{+}$the parameters $a_{1}$ and $b_{1}$ are the same in both long-time limits. Thus, soliton 1 has the same form in both long-time limits but undergoes a phase shift due to interaction with soliton 2.

The asymptotic limits of soliton 2 are similar to those for soliton 1 except that the relative change in sign in (22) reverses the calculations, that is,

$$
\mathbf{q}_{2, n}^{-}=\mathbf{c}_{2}^{-} \hat{q}_{2, n}^{-} \quad \text { and } \quad \mathbf{r}_{2, n}^{-}=\mathbf{d}_{2}^{-} \hat{q}_{2, n}^{-*}
$$

where $\mathbf{c}_{2}^{-}$has the form (27a) and $\mathbf{d}_{2}^{-}$has the form (27b) with the indices 1 and 2 exchanged. The scalar function $\hat{q}_{2, n}^{-}$is a one-soliton solution of IDNLS with $a=a_{2}, b=n_{2}$ and $\theta=-\delta_{2}^{-}$where $\delta_{2}^{-}$is equal to $\delta_{1}^{+}$ with the indices 1 and 2 exchanged. Similarly,

$$
\mathbf{q}_{2, n}^{+}=\mathbf{c}_{2}^{+} \hat{q}_{2, n}^{+} \quad \text { and } \quad \mathbf{r}_{2, n}^{+}=\mathbf{d}_{2}^{+} \hat{q}_{2, n}^{+*}
$$

where $\mathbf{c}_{2}^{+}$has the form (24a) and $\mathbf{d}_{2}^{+}$has the form (24b) with the indices 1 and 2 exchanged and $\hat{q}_{2, n}^{+}$is a one-soliton solution of IDNLS with $a=a_{2}, b=n_{2}$ and $\theta=-\delta_{2}^{+}$where $\delta_{2}^{+}$is equal to $\delta_{1}^{-}$with the indices 1 and 2 exchanged.

In order to construct a solution such that

$$
\mathbf{r}_{j, n}^{-}=-\mathbf{q}_{j, n}^{-*}
$$

for $j=1,2$ it is necessary and sufficient that

$$
\begin{aligned}
& \bar{\gamma}_{1}=\gamma_{1}^{*} \\
& \overline{\boldsymbol{\gamma}}_{2}=\frac{1}{\left\|\boldsymbol{\gamma}_{1}\right\|^{2}}\left(\begin{array}{ll}
\left|\gamma_{1}^{(1)}\right|^{2}+\left|e^{-h a_{1}} \gamma_{1}^{(2)}\right|^{2} & \left(1-e^{-2 h a_{1}}\right) \gamma_{1}^{(1) *} \gamma_{1}^{(2)} \\
\left(1-e^{-2 h a_{1}}\right) \gamma_{1}^{(1)} \gamma_{1}^{(2)^{*}} & \left|e^{-h a_{1}} \gamma_{1}^{(1)}\right|^{2}+\left|\gamma_{1}^{(2)}\right|^{2}
\end{array}\right) \gamma_{2}^{*}
\end{aligned}
$$

For this choice, $\chi$ and $\bar{\gamma}_{j}^{T} \boldsymbol{\gamma}_{j}$ for $j=1,2$ are real and positive as we required. However, if (28a 28b) hold then

$$
\mathbf{r}_{j, n}^{+} \neq-\mathbf{q}_{j, n}^{+*}
$$

for $j=1,2$. In fact, $\left\|\mathbf{r}_{j, n}^{+}-\left(-\mathbf{q}_{j, n}^{+*}\right)\right\|=O(h)$. An alternative is to set

$$
\bar{\gamma}_{1}=\gamma_{1}^{*} \quad \text { and } \quad \bar{\gamma}_{2}=\gamma_{2}^{*} .
$$

Then, if (30) holds, $\chi$ and $\overline{\boldsymbol{\gamma}}_{j}^{T} \boldsymbol{\gamma}_{j}$ - for $j=1,2$-are real and positive and

$$
\mathbf{r}_{1, n}^{-}=-\mathbf{q}_{1, n}^{-*}, \quad \mathbf{r}_{2, n}^{+}=-\mathbf{q}_{2, n}^{+*}
$$

but

$$
\left\|\mathbf{r}_{1, n}^{+}-\left(-\mathbf{q}_{1, n}^{+*}\right)\right\|=O(h)=\left\|\mathbf{r}_{2, n}^{-}-\left(-\mathbf{q}_{2, n}^{-*}\right)\right\| .
$$

In contrast, recall that the solutions of the scalar IDNLS converge to solutions of the scalar NLS with $O\left(h^{2}\right)$ convergence. This unavoidable asymmetry of the system (5a-5b) makes it less desirable as a discrete approximation of VNLS. Hence, we turn our attention to the symmetric system. 


\section{Symmetric Discrete Vector NLS}

The symmetric system (4) is the natural vector generalization of IDNLS (3). We now describe some important symmetries of the symmetric system. In analogy with the scalar case (3), the symmetric system can be thought of as the reduction of the system

$$
\begin{aligned}
i \frac{\mathrm{d}}{\mathrm{d} t} \mathbf{q}_{n} & =\frac{1}{h^{2}}\left(\mathbf{q}_{n-1}-2 \mathbf{q}_{n}+\mathbf{q}_{n+1}\right)-\mathbf{r}_{n}^{T} \mathbf{q}_{n}\left(\mathbf{q}_{n-1}+\mathbf{q}_{n+1}\right) \\
-i \frac{\mathrm{d}}{\mathrm{d} t} \mathbf{r}_{n} & =\frac{1}{h^{2}}\left(\mathbf{r}_{n-1}-2 \mathbf{r}_{n}+\mathbf{r}_{n+1}\right)-\mathbf{q}_{n}^{T} \mathbf{r}_{n}\left(\mathbf{r}_{n-1}+\mathbf{r}_{n+1}\right)
\end{aligned}
$$

obtained by letting $\mathbf{r}_{n}=-\mathbf{q}_{n}^{*}$, which is the symmetry that is broken by the asymmetric system.

Under the reduction

$$
\mathbf{q}_{n}=\mathbf{c} \hat{q}_{n}
$$

where $\|\mathbf{c}\|=1$, the symmetric system reduces to the scalar IDNLS (3). Furthermore, if $\mathbf{q}_{n}$ satisfies the symmetric system, then so does $\mathbf{U} \mathbf{q}_{n}$ where $\mathbf{U}$ is a unitary matrix (this symmetry is a discrete form of eq. (7)). Therefore, the symmetric discretization retains the reductions and symmetries of the asymmetric system and has the additional symmetry $\mathbf{r}_{n}=-\mathbf{q}_{n}^{*}$.

Despite the above-mentioned symmetries, there is, to date, no known associated linear operator pair for the symmetric system. Without such a pair, the system (31a $31 \mathrm{~b}$ ) cannot be solved by IST. The question remains, however, whether the symmetric system is integrable. In the absence of IST, the existence of multi-soliton solutions provides strong circumstantial evidence of integrability.

In order to determine whether there are solitary waves which interact elastically- i.e. solitons, we first identify a solitary-wave solution of the symmetric system: a solution of the form (32) where the scalar $\hat{q}_{n}$ is a one-soliton solution of IDNLS (as in eq. (6)) is a solitary-wave solution of the symmetric system. Note that such a solution can have any polarization $\mathbf{c}$ subject only to the constraint that $\|\mathbf{c}\|^{2}=1$. In order determine whether these vector solitary waves interact exactly, we use both direct and numerical methods.

\subsection{Soliton solutions by Hirota's Method}

Soliton solutions of the symmetric system can be found by Hirota's method. For concreteness, we consider the case where $\mathbf{q}_{n}$ has two components $(N=2)$. Under the independent variable transformation,

$$
\mathbf{q}_{n}=\frac{\mathbf{g}_{n}}{f_{n}}
$$

where $\mathbf{g}$ is a vector and $f_{n}$ is a scalar, solutions of the bilinear equations

$$
\begin{gathered}
i h^{2} D_{t} f_{n} \cdot \mathbf{g}_{n}=f_{n-1} \mathbf{g}_{n+1}-2 f_{n} \mathbf{g}_{n}+f_{n+1} \mathbf{g}_{n-1} \\
f_{n+1} f_{n-1}-f_{n}^{2}=h^{2}\left\|\mathbf{g}_{n}\right\|^{2}
\end{gathered}
$$

are solutions of the symmetric system.

The following solution of the bilinear equations yields a two-soliton solution:

$$
\begin{aligned}
f & =1+e^{\eta_{1, n}+\eta_{1, n}^{*}}+e^{\eta_{2, n}+\eta_{2, n}^{*}}+\left|B_{1}\right|^{2} e^{\eta_{1, n}+\eta_{1, n}^{*}+\eta_{2, n}+\eta_{2, n}^{*}} \\
g^{(1)} & =\frac{1}{h}\left(e^{h p_{1}}-e^{-h p_{1}^{*}}\right) e^{\eta_{1, n}}\left(1+B_{1} e^{\eta_{2, n}+\eta_{2, n}^{*}}\right) \\
g^{(2)} & =\frac{1}{h}\left(e^{h p_{2}}-e^{-h p_{2}^{*}}\right) e^{\eta_{2, n}}\left(1+B_{2} e^{\eta_{1, n}+\eta_{1, n}^{*}}\right)
\end{aligned}
$$

where $\eta_{j, n}$ and $p_{j}$ are as in eq. (15) and 


$$
B_{1}=\frac{\left(e^{h p_{1}}-e^{h p_{2}}\right)\left(e^{h p_{1}}+e^{h p_{2}^{*}}\right)}{\left(e^{h\left(p_{1}+p_{2}\right)}+1\right)\left(e^{h\left(p_{1}+p_{2}^{*}\right)}-1\right)}, \quad B_{2}=-\frac{\left(e^{h p_{1}}-e^{h p_{2}}\right)\left(e^{h p_{1}^{*}}+e^{h p_{2}}\right)}{\left(e^{h\left(p_{1}+p_{2}\right)}+1\right)\left(e^{h\left(p_{1}^{*}+p_{2}\right)}-1\right)}
$$

In order to see that the above solution indeed gives a two-soliton solution, consider the long-time limits. We calculate the long-time limits of the Hirota form of the solution (34a-34d) by the same approach as was used for the asymmetric case. In the forward $(+)$ and backward (-) long-time limits, the solution a (34a-34d) asymptotically approaches the form

$$
\mathbf{q}_{n} \sim \mathbf{q}_{1, n}^{ \pm}+\mathbf{q}_{2, n}^{ \pm}
$$

as $t \rightarrow \pm \infty$.

The long-time limits for soliton 1 are

$$
\begin{aligned}
& \mathbf{q}_{1, n}^{-}=\left(\begin{array}{c}
e^{i \phi_{1}^{-}} \\
0
\end{array}\right) \frac{\sinh \left(a_{1} h\right)}{h} e^{i \beta_{1, n}} \operatorname{sech}\left(\alpha_{1, n}\right) \\
& \mathbf{q}_{1, n}^{+}=\left(\begin{array}{c}
e^{\phi_{1}^{+}} \\
0
\end{array}\right) \frac{\sinh \left(a_{1} h\right)}{h} e^{i \beta_{1, n}} \operatorname{sech}\left(\alpha_{1, n}+\log \left|B_{1}\right|\right)
\end{aligned}
$$

where

$$
\phi_{1}^{-}=h b_{1}, \quad \phi_{1}^{+}=h b_{1}+\arg B_{1}
$$

and $\alpha_{1, n}, \beta_{1, n}$ are as in (17a-17b). In both limits, this is a solution of the form $\mathbf{q}_{1, n}^{ \pm}=\mathbf{c}_{1}^{ \pm} \hat{q}_{1, n}$ where $\hat{q}_{1, n}^{ \pm}$is a one-soliton solution of IDNLS (as in (6)). Note that the polarization, $\mathbf{c}_{1}^{ \pm}$, is such that $\left|c_{1}^{(1) \pm}\right|^{2}=1$ (and, therefore, $\left|c_{1}^{(2) \pm}\right|^{2}=0$ since $\left.\left\|\mathbf{c}_{1}^{ \pm}\right\|^{2}=1\right)$.

The long-time limits for soliton 2 are

$$
\begin{aligned}
& \mathbf{q}_{2, n}^{-}=\left(\begin{array}{c}
0 \\
e^{i \phi_{2}^{-}}
\end{array}\right) \frac{\sinh \left(a_{2} h\right)}{h} e^{i \beta_{2, n}} \operatorname{sech}\left(\alpha_{2, n}+\log \left|B_{1}\right|\right) \\
& \mathbf{q}_{2, n}^{+}=\left(\begin{array}{c}
0 \\
e^{i \phi_{2}^{+}}
\end{array}\right) \frac{\sinh \left(a_{2} h\right)}{h} e^{i \beta_{2, n}} \operatorname{sech}\left(\alpha_{2, n}\right)
\end{aligned}
$$

where

$$
\phi_{2}^{-}=h b_{2}+\arg B_{2}, \quad \phi_{2}^{+}=h b_{2}
$$

and $\alpha_{2, n}, \beta_{2, n}$ are as in (17a $\left.17 \mathrm{~b}\right)$ with $a_{2}$ and $b_{2}$ replacing $a_{1}$ and $b_{1}$ respectively. In both the forward and backward long-time limit, this is a solution of the form $\mathbf{c}_{2}^{ \pm} \hat{q}_{2, n}^{ \pm}$where $\left|c_{2}^{(2) \pm}\right|^{2}=1$ (and, therefore, $\left|c_{2}^{(2) \pm}\right|^{2}=0$ since $\left.\left\|\mathbf{c}_{2}^{ \pm}\right\|^{2}=1\right)$.

The solution (35a $35 \mathrm{~b}$, 36a $36 \mathrm{~b}$ ) is not the most general two-soliton interaction because

$$
\left|\mathbf{c}_{1}^{-} \cdot \mathbf{c}_{2}^{-}\right|=\left|\mathbf{c}_{1}^{+} \cdot \mathbf{c}_{2}^{+}\right|=0 .
$$

The two-soliton solution $35 \mathrm{a}-35 \mathrm{~b}$, $36 \mathrm{a}-36 \mathrm{~b})$ can be multiplied by a unitary matrix to obtain a two-soliton solution with any $\mathbf{c}_{1}^{-}, \mathbf{c}_{1}^{+}$such that $\left|\mathbf{c}_{1}^{-} \cdot \mathbf{c}_{2}^{-}\right|=0$ (under any such transformation, the condition $\left|\mathbf{c}_{1}^{+} \cdot \mathbf{c}_{2}^{+}\right|=0$ will still hold). Thus, these two-soliton solutions are a constrained class of two-soliton solutions where (37) is the constraint. 
More generally, Hirota's method can be used to derive solutions of the symmetric system with more than two solitons. These solutions can be represented as combinations of Pfaffians (these formulae are quite technical; they are presented in a separate publication [14). The $M$-soliton solutions derived in this manner are constrained such that either

$$
\left|\mathbf{c}_{j}^{-} \cdot \mathbf{c}_{k}^{-}\right|=1
$$

or

$$
\left|\mathbf{c}_{j}^{-} \cdot \mathbf{c}_{k}^{-}\right|=0
$$

for all $j, k=1, \ldots, M$. Moreover, the above satisfy

$$
\left|\mathbf{c}_{j}^{-} \cdot \mathbf{c}_{j}^{+}\right|=1
$$

for all $j=1, \ldots, M$. These multi-soliton solutions are reminiscent of the special case of soliton interactions in the PDE discussed at the end of section 2.2: conditions (38a $38 \mathrm{~b}$ ) and eq. (39) for the discrete symmetric system are the counterparts of (9a-9b) and eq. (10) in the PDE. To date, there is no known analytic formula for a general multi-soliton solution of the symmetric system such that $0<\left|\mathbf{c}_{j}^{-} \cdot \mathbf{c}_{k}^{-}\right|<1$ and $\left|\mathbf{c}_{j}^{-} \cdot \mathbf{c}_{j}^{+}\right|<1$.

\subsection{Numerical Simulation of Soliton Interactions}

The individual solitary waves of the symmetric system may have any polarization. In the PDE (2) such solitary waves interact as solitons. That is, there are vector multi-soliton solutions of VNLS with asymptotic polarizations $\mathbf{c}_{j}^{ \pm}$such that $0<\left|\mathbf{c}_{j}^{-} \cdot \mathbf{c}_{k}^{-}\right|<1$ and $\left|\mathbf{c}_{j}^{-} \cdot \mathbf{c}_{j}^{+}\right|<1$. In the absence of analytical formulae in the discrete symmetric system for these general multi-soliton interactions, we investigated the collision of solitary waves by numerical simulation.

In the simulations, the initial conditions were taken to be of the form

$$
\mathbf{q}_{n}^{-}=\mathbf{q}_{1, n}^{-}+\mathbf{q}_{2, n}^{-}
$$

where $\mathbf{q}_{j, n}^{-}=\mathbf{c}_{j}^{-} \hat{q}_{j, n}^{-}$and $\hat{q}_{j, n}^{-}$is of the form (6). Then, the symmetric system was integrated in time by an adaptive Runge-Kutta-Merson routine (from the NAG library) until the peaks were again well-separated (see Figure 1 for an example). The separation of peaks in the initial and final conditions makes these conditions comparable to, respectively, the backwards and forwards long-time limits. The solitary-wave interactions simulated in this manner comprised initial conditions in which $0<\left|\mathbf{c}_{j}^{-} \cdot \mathbf{c}_{k}^{-}\right|<1$. Visually, in Figure 1 (and in other simulations) the solitary waves appear to interact without any radiation. We confirmed this finding by quantitative comparison of the solitary waves at the final time and exact solitary waves with the same height and speed parameters as the initial data.

The error at the final time was defined separately for each solitary wave by

$$
\Delta_{j}=\frac{1}{A_{j}} \max _{n \in \Omega_{j}}\left\|\mathbf{q}_{j, n}^{f}-\mathbf{q}_{j, n}^{+}\right\|
$$

where:

$\mathbf{q}_{j, n}^{f}$ is the numerical data at the final time;

$\mathbf{q}_{j, n}^{+}$is a solitary wave with the same amplitude and speed parameters- $a_{j}$ and $b_{j}$-as the initial data, $\mathbf{q}_{j, n}^{-}$;

$A_{j}=\frac{\sinh \left(a_{j} h\right)}{h}$ is the amplitude of the exact solitary wave with amplitude parameter $a_{j}$; 
$\Omega_{j}$ is the set of points containing the main contribution of the $j$-th solitary wave- i.e. $\Omega_{j}=\left\{n:\left\|\mathbf{q}_{n, j}^{f}\right\|>\epsilon\right\}$ for $\epsilon$ small compared to $\max _{j=1,2} A_{j}$.

The resulting errors were small (see Table 1 for an example). Furthermore, when the user-supplied error bound in the adaptive integration scheme was decreased, the errors, $\Delta_{j}$, decreased proportionally. Therefore, the differences between the final wave forms obtained by numerical simulation and exact solitary waves are accounted for by errors in the time integration. These results, strongly suggest that the solitary waves interact elastically- i. e. the solitary waves are solitons.

In the numerical simulations described in Table 1, and in other experiments we considered initial data such that $0<\left|\mathbf{c}_{1}^{-} \cdot \mathbf{c}_{2}^{-}\right|<1$, the general case of the soliton interaction for which there is no known analytical solution. In the PDE (2), such conditions result in the shift of the polarizations of the individual vector solitons- i.e. $\left|\mathbf{c}_{j}^{-} \cdot \mathbf{c}_{j}^{+}\right|<1, j=1,2$. The simulations described in Table 1 , consistent with other numerical experiments, show the same distinctive vector soliton behavior for the discrete symmetric system.

The mechanism of the more general elastic soliton interactions observed in the symmetric system remains to be explained analytically. More generally, proof that the symmetric system is integrable remains as an important open problem.

\section{Acknowledgments}

This effort was sponsored in part by the Air Force Office of Scientific Research, Air Force Materials Command, USAF, under grant number F49620-97-1-0017, by the Office of Naval Research, USN, under grant number N00014-94-1-0915 and the National Science Foundation under grant number DMS-9703850. The US Government is authorized to reproduce and distribute reprints for governmental purposes notwithstanding any copyright notation thereon. The views and conclusions contained herein are those of the authors and should not be interpreted as necessarily representing the official policies or endorsements, either expressed or implied, of the Air Force Office of Scientific Research or the US Government. One of the authors (YO) is grateful to Dr. S. Tsujimoto for valuable discussions and also acknowledges the financial support of the Japan Ministry of Education through the Foreign Study Program.

\section{References}

[1] M. J. Ablowitz and H. Segur. Solitons and the Inverse Scattering Transfom. Number 4 in SIAM Studies in Applied Mathematics. SIAM, 1981.

[2] V. E. Zakharov and A. B. Shabat. Exact theory of two-dimensional self-focusing and one-dimensional self-modulation of waves in nonlinear media. Soviet Physics JETP, 34:62-69, 1972.

[3] S. V. Manakov. On the theory of two-dimensional stationary self-focusing of electromagnetic waves. Soviet Physics JETP, 38(2):248-253, 1974.

[4] C. R. Menyuk. Nonlinear pulse propagation in birefringent optical fibers. IEEE Journal of Quantum Electronics, QE-23(2):174-176, February 1987.

[5] S. G. Evangelides, L. F. Mollenauer, J. P. Gordon, and N. S. Bergano. Polarization multiplexing with solitons. Journal of Lightwave Technology, 10(1):28-35, January 1992.

[6] C. R. Menyuk. Pulse propagation in an elliptically birefringent Kerr medium. IEEE Journal of Quantum Electronics, 25(12):2674-2682, December 1989.

[7] M. J. Ablowitz and J. F. Ladik. A nonlinear difference scheme and inverse scattering. Studies in Applied Mathematics, 55:213-229, 1976.

[8] Ch. Claude, Yu. S. Kishvar, O. Kluth, and K. H. Spatscheck. Moving modes in localized nolnlinear lattices. Physical Review B, 47(21):14228-14232, June 1993. 
[9] J. C. Eilbeck, P. S. Lombdahl, and A. C. Scott. The discrete self-trapping equation. Phyisica, 16 D:318-338, 1985.

[10] A. R. Its, A. G. Izergin, V. E. Korepin, and N. A. Slavnov. Temperature correlations of quantum spins. Physical Review letters, 70(11):1704-1706, March 1993.

[11] A. A. Vakhnenko and Yu. B. Gaididei. On the motion of solitons in discrete molecular chains. Theoretical and Mathematical Physics, 68(3):873-880, March 1987.

[12] M. J. Ablowitz and P. A. Clarkson. Solitons Nonlinear Evolution Equations and Inverse Scattering. Number 149 in London Mathematical Society Lecture Note Series. Cambridge University Press, 1991.

[13] Y. B. Suris. A discrete-time Garnier system. Physics Letters. A, 189(4):281-289, 1994.

[14] Y. Ohta. Pfaffian solutions for coupled discrete nonlinear schrödinger equation. Chaos, Solitons and Fractals, to appear. Proceedings of Brussels Meeting II: Integrability and Chaos in Discrete Systems (Brussels, 2-6 July 1997). 
$t=-8.46$
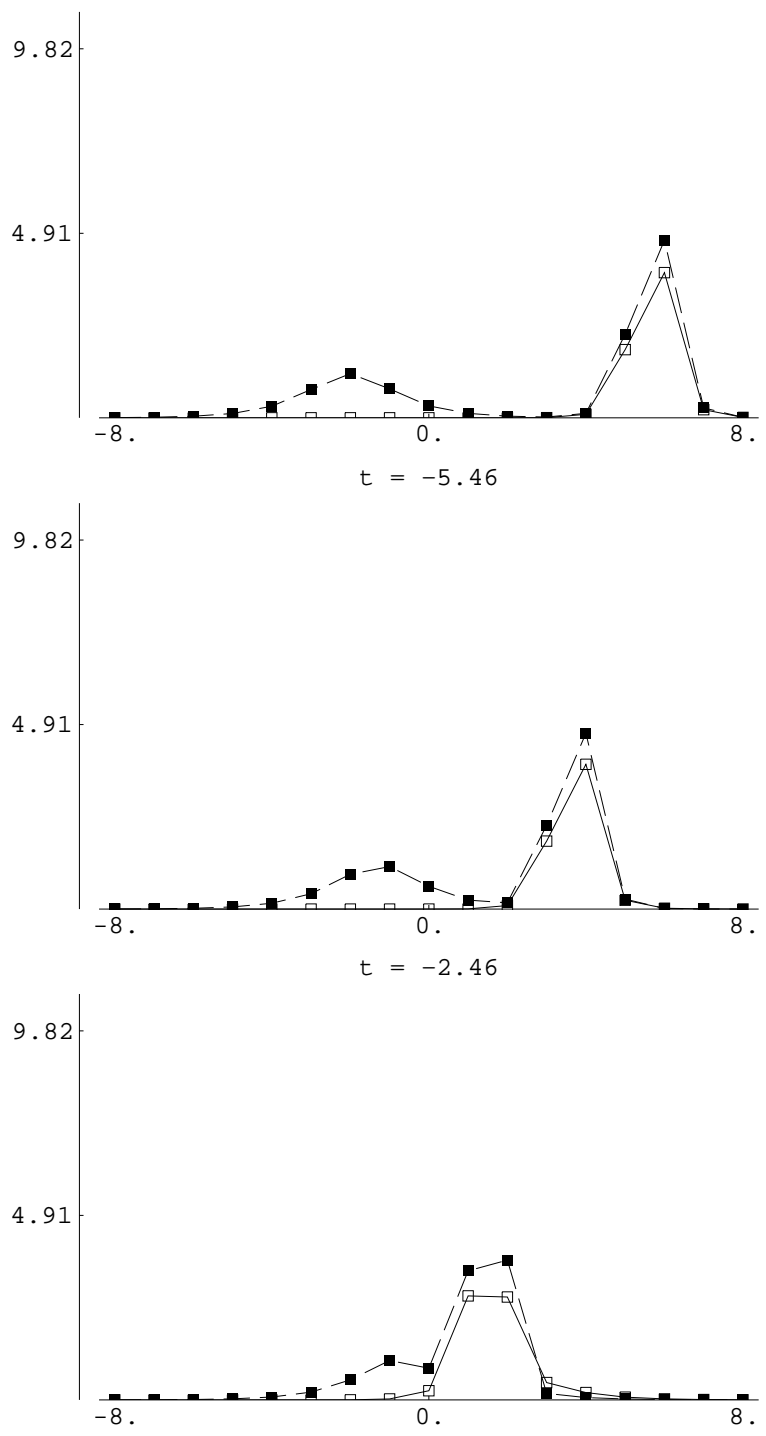
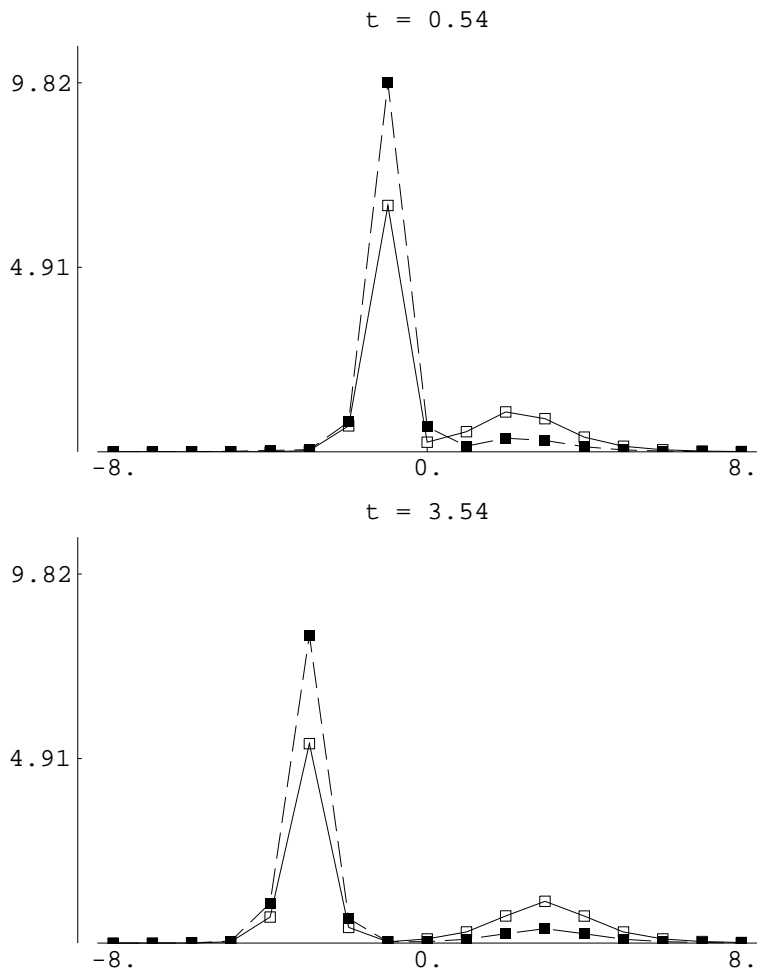

$t=6.54$

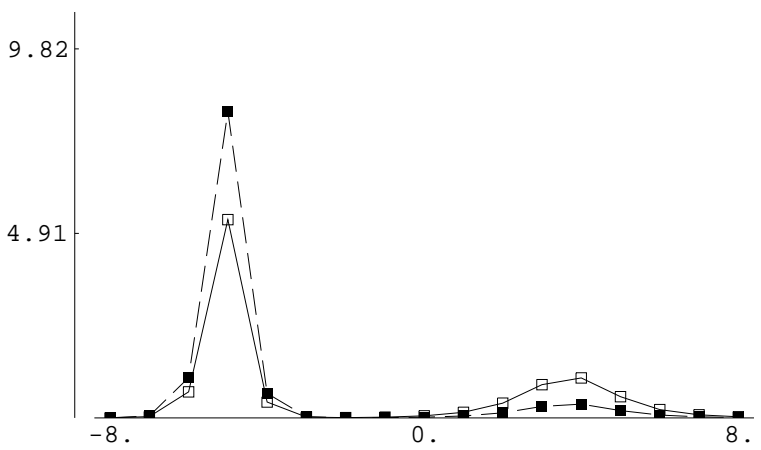

Figure 1: Two-soliton interaction for the symmetric system. The filled boxes are $\left|q_{n}^{(1)}\right|$ and the open boxes are $\left|q_{n}^{(2)}\right|$. Increasing time is read down column-wise. Soliton 1 is on the left and Soliton 2 is on the right at $t=-8.46$. They are reversed at $t=6.54$. The soliton parameters are: $a_{1}=1, a_{2}=3, b_{1}=.1, b_{2}=-.1$. The polarization vectors are: $\mathbf{c}_{1}^{-}=(1,0), \mathbf{c}_{2}^{-}=(.60, .80)$, at $t=-8.46$, and $\mathbf{c}_{1}^{+}=\left(.33 e^{i .23 \pi}, .95 e^{i .12 \pi}\right)$, $\mathbf{c}_{2}^{+}=\left(.84 e^{-i .02 \pi}, .54 e^{-i .01 \pi}\right)$ at $t=6.54$. This is a typical two-soliton interaction where $0<\left|\mathbf{c}_{1}^{-} \cdot \mathbf{c}_{2}^{-}\right|=.6<1$, $\left|\mathbf{c}_{1}^{-} \cdot \mathbf{c}_{1}^{+}\right|=.204<1$ and $\left|\mathbf{c}_{2}^{-} \cdot \mathbf{c}_{2}^{+}\right|=.993<1$. 


\begin{tabular}{|c|c|c|c|c|}
\hline $\mathbf{c}_{2}^{-}$ & $\left|\mathbf{c}_{1}^{-} \cdot \mathbf{c}_{1}^{+}\right|$ & $\left|\mathbf{c}_{2}^{-} \cdot \mathbf{c}_{2}^{+}\right|$ & $\log _{10} \Delta_{1}$ & $\log _{10} \Delta_{2}$ \\
\hline$(0.0,1.0)$ & 1.000 & 1.000 & -7.57 & -7.57 \\
$(0.2, .98)$ & 0.575 & 0.575 & -7.54 & -7.54 \\
$(0.4, .92)$ & 0.492 & 0.492 & -7.52 & -7.52 \\
$(0.6,0.8)$ & 0.624 & 0.624 & -7.58 & -7.58 \\
$(0.8,0.6)$ & 0.806 & 0.806 & -7.56 & -7.56 \\
$(1.0,0.0)$ & 1.000 & 1.000 & -7.57 & -7.57 \\
\hline
\end{tabular}

\begin{tabular}{|c|c|c|c|c|}
\hline $\mathbf{c}_{2}^{-}$ & $\left|\mathbf{c}_{1}^{-} \cdot \mathbf{c}_{1}^{+}\right|$ & $\left|\mathbf{c}_{2}^{-} \cdot \mathbf{c}_{2}^{+}\right|$ & $\log _{10} \Delta_{1}$ & $\log _{10} \Delta_{2}$ \\
\hline$(0.0,1.0)$ & 1.000 & 1.000 & -8.92 & -6.21 \\
$(0.2, .98)$ & 0.900 & 0.999 & -7.75 & -6.12 \\
$(0.4, .92)$ & 0.617 & 0.996 & -7.41 & -6.06 \\
$(0.6,0.8)$ & 0.204 & 0.993 & -7.27 & -6.07 \\
$(0.8,0.6)$ & 0.396 & 0.994 & -7.37 & -6.12 \\
$(1.0,0.0)$ & 1.000 & 1.000 & -7.61 & -6.25 \\
\hline
\end{tabular}

Table 1: Two-Soliton interaction for the symmetric system (何). The soliton amplitude parameters are $a_{1}=1$ and $a_{2}=1$ or $a_{2}=3$ as it is given in each of the tables. For these values of $a_{j}$, the soliton width is comparable to the grid size and the solution is not "close" to the continuum limit. The soliton speed parameters, $b_{1}=.1$ and $b_{2}=-.1$, are such that the solitons move slowly relative to one another thereby increasing the strength of the interaction. The polarizations before interaction are $\mathbf{c}_{1}^{-}=(1,0)$ and $\mathbf{c}_{2}^{-}$as it is given in the tables. The polarizations after interaction are $\mathbf{c}_{1}^{+}$and $\mathbf{c}_{2}^{+} . \Delta_{j}$ where $j=1,2$, is the difference, as given by eq. (40), between the numerical solution at the final time and an exact solitary wave. The values $\left|\mathbf{c}_{j}^{-} \cdot \mathbf{c}_{j}^{+}\right|<1$ indicate that the polarization vectors are shifted by the soliton interaction. The small errors, $\Delta_{j}$, show that the solitary waves interact nearly elastically with the measured deviation accounted for by error in the numerical time integration. 